\title{
Surgical Management of Clostridium difficile Infection: The Role of Colectomy
}

\author{
Matthew J. Delano ${ }^{1}$ and Joseph Cuschieri ${ }^{1,2}$
}

\begin{abstract}
Management of Clostridium difficile infections is usually accomplished through appropriate antimicrobial therapy. However, in patients that do not respond to this therapy, rapid and potentially lethal progressive organ dysfunction care occurs. Although supportive care and continued antimicrobial therapy is important, surgical therapy is critical to eradication of the inflammatory process and reversal of the dysregulated immunity associated with severe $C$. difficile infections. In the following paper, the role of colectomy is reviewed.
\end{abstract}

$S^{\mathrm{s}}$ IXTY YEARS after the first report of Staphylococcus enterocolitis, we are in the midst of a Clostridium diffcile infection (CDI) resurgence [1] and its accompanying morbidity and mortality rates [2]. Our knowledge of this pathogen has evolved considerably over the last half century. Clostridium difficile is a spore-forming, anaerobic, grampositive bacterium. This microbe is responsible for gastrointestinal infections that range in severity from mild colitis to toxic megacolon and death. Clostridium difficile remains the leading cause of healthcare-associated diarrhea and now rivals methicillin-resistant Staphylococcus aureus (MRSA) as the most common organism to cause healthcare-associated infection in the United States [3,4]. In addition, infections caused by $C$. difficile have been reported in patients previously believed to be at "low risk," lacking the typical risk factors for infection, such as prior antimicrobial therapy or extended stay in a healthcare facility $[2,4]$. Accompanying the increase in prevalence is an associated increase in disease severity [5], no longer allowing CDI to be brushed aside as an indolent infection. Many recent cases of CDI have resulted in substantial morbidity and mortality rates, causing healthcare providers to search for a more integrated and streamlined management scheme to more optimally transition from medical management to surgical intervention [6].

The newfound CDI prevalence and disease severity appear to be multi-factorial, but can only be partially explained by recent advances in CDI pathophysiology. The primary virulence factors of $C$. difficile are Toxin A (TcdA) and Toxin B $(\mathrm{TcdB})$. These toxins are potent cytotoxic enzymes that damage the human colonic mucosa, causing the traditional symptoms of CDI. A binary toxin called cytolethal distending toxin (CDT) has been identified in approximately $6 \%$ of $C$. difficile isolates and is a characteristic feature of a hypervirulent $C$. difficile strain. This toxin is hypothesized to potentiate the toxicity of TcdA and TcdB, leading to more severe disease [7]. The emergence of a novel, hypervirulent strain of C. difficile has now been reported across the United States, Canada, and Europe [5,7,8]. This strain of $C$. difficile has been characterized as toxinotype III, North American pulsedfield gel electrophoresis (PFGE) type 1, and polymerase chain reaction (PCR)-ribotype 027 (NAP1/027). This new strain appears to produce greater amounts of Toxins A and B and has a deletion in the $t c d C$ gene, which potentially down regulates toxin production. In addition, this hypervirulent strain exhibits increased sporulation in comparison to nonvirulent strains of $C$. difficile, which may contribute to the widespread observed disease severity [7].

Although the number of CDI cases is increasing, the surgical intervention rate appears to be constant, causing many healthcare providers concern. According to the Nationwide Inpatient Sample of the Healthcare Cost and Utilization Project (HCUP), the total number of patients assigned the International Classification of Diseases, Ninth Revision (ICD-9) code for CDI (008.45) in acute care facilities has increased from 138,954 in 2000 to nearly 350,000 in 2008, whereas the number of surgical interventions has remained constant $[9,10]$. The most well-known risk factors for the development of CDI are the administration of antibiotics, inappropriate use of broad-spectrum agents, and lack of appropriate streamlining based on culture/susceptibility data.

\footnotetext{
${ }^{1}$ Department of Surgery, University of Michigan, Ann Arbor, Michigan.

${ }^{2}$ Department of Surgery, University of Washington, Seattle, Washington.

Presented at the 30th Annual Meeting of the Surgical Infection Society, Las Vegas, Nevada, April 17-20, 2010.
} 
Almost every antibiotic has been implicated in the development of CDI, but the most commonly cited agents include clindamycin, cephalosporins, and fluoroquinolones. Advanced age is also associated with increased risk for CDI, as is a prolonged stay in a healthcare facility such as an acute care institution or a long-term care facility. In addition, high severity of illness is also a notable risk factor for CDI [6,9,11-14].

Other factors have been linked to CDI with varying levels of supporting evidence. Patients with inflammatory bowel disease are at greater risk than the general population for acquiring CDI [12]. This association may be because of the multiple courses of antibiotics necessary for frequent disease flares in addition to the use of immunosuppressive agents to maintain disease remission. Gastrointestinal surgery has also been linked to CDI, although this factor is not a confirmed risk factor for disease. There also has been a considerable amount of interest in proton pump inhibitors (PPIs) and resulting gastric acid suppression as a causative factor of CDI [11]. Proton pump inhibitors have been implicated in the development of community-acquired CDI, although conflicting data exist to date. A high severity of illness and immunosuppression has also been attributed to increased risk of CDI in some cases [14]. Certain factors can also put patients at risk for CDI recurrence and even poorer outcomes. These factors include advanced age $>65 \mathrm{y}$; administration of antibiotics after initial treatment of CDI; prolonged hospitalization or stay in a long-term care facility; a defective immune response to Toxin $\mathrm{A}$; and gastric acid suppression [13,15-17].

Confirmation of CDI can be challenging considering the multitude of diagnostic tests available and their individual and collective lack of specificity and sensitivity for predicting clinically substantial infection. Enzyme immunoassay (EIA), tissue culture cytotoxicity, glutamate dehydrogenase (GDH), polymerase chain reaction (PCR), and stool culture for C. difficile are all efficacious. There are clearly benefits to any of these diagnostic modalities depending on the clinical situation [6].

The basic principles of CDI therapy include discontinuing the offending antimicrobial agent, sending a stool specimen early for $C$. difficile testing, initiating CDI therapy empirically or following confirmation of diagnosis, and monitoring for symptom resolution or progression with awareness of potential for recurrence after treatment discontinuation. Therapy may include pharmacotherapy with agents such as vancomycin (the only U.S. Food and Drug Administration [FDA]-approved treatment for CDI) or metronidazole, or supportive care [6]. According to the Society for Healthcare Epidemiology of America (SHEA)/Infectious Diseases Society of America (IDSA) guidelines, mild to moderate disease is defined as white blood cell count (WBC) $<1500$ cells/mcL or serum creatinine ( $\mathrm{SCr}$ ) concentration $<1.5$ times the pre-morbid concentration. In these patients, the recommended therapy is metronidazole $500 \mathrm{mg}$ three times per day per oral for 10-14 d.

Alternately, severe disease is defined as WBC $\geq 1500$ cells/ $\mathrm{mcL}$ or $\mathrm{SCr}$ concentration $\geq 1.5$ times the pre-morbid concentration. The recommended treatment for severe CDI is vancomycin $125 \mathrm{mg}$ four times per day PO for 10-14 d. Severe, complicated disease is defined as hypotension or shock, ileus, or toxic megacolon. These patients should receive vancomycin $500 \mathrm{mg}$ four times per day $\mathrm{PO}$ or by nasogastric tube plus metronidazole $500 \mathrm{mg}$ IV every $8 \mathrm{~h}$. It is important to note that the vancomycin dose in this setting is based on original, empiric studies; it is not clear that $500 \mathrm{mg}$ is necessary [6]. Lastly, a surgical consult should be obtained in all patients with complicated CDI and operative intervention considered in all patients with hypotension requiring vasopressor therapy, clinical signs of sepsis and organ dysfunction, mental status changes, WBC $>50,000$ cells $/ \mathrm{ml}$, lactate $\geq 5 \mathrm{mmol} / \mathrm{L}$, or failure to improve on medical therapy for $5 \mathrm{~d}$ [6]. Once severe, complicated CDI is recognized and evaluated for potential surgical intervention, it should be note that colectomy may be lifesaving, but is associated with increased risk of death if WBC is $>50,000$ cells $/ \mathrm{mcL}$ and lactate is $>5 \mathrm{mg} / \mathrm{dL}$. Indications of severe, complicated disease course include the following: Elevated and rising WBC, elevated $\mathrm{SCr}$ concentration, elevated serum lactate, clinical and radiographic evidence of severe ileus, and impending toxic megacolon. Vancomycin per rectum should also be considered if ileus is severe or operative approaches are not suitable $[6,18]$.

Surgical options for severe CDI intervention include segmental partial colon resection, subtotal colectomy with end ileostomy (TCEI), and diverting loop ileostomy with colonic irrigation [19], each approach having its benefits and disadvantages. Outcome following colectomy with ileostomy versus partial colectomy for severe CDI is difficult to interrupt based on limited data. Most authors favor TCEI over partial colectomy alone [19]. Following colectomy for severe CDI in one study, patients with virulent fulminant CDI experienced a reduced mortality rate from $58 \%$ to $34 \%$ [20]. Some of the factors associated with improved mortality rates following colectomy for severe CDI include age $>75 \mathrm{y}$, absence of immune suppression, shock requiring vasopressor amines, WBC between 19,900 and 49,900 cells/mcL, and peak lactate $2.2 \mathrm{mmol} / \mathrm{L}$ to $4.9 \mathrm{mmol} / \mathrm{L}$ [20]. Conversely, proposed factors associated with colectomy failure include delayed surgical intervention occurring too late in the course of the disease, incorrect patient selection because of a lack of clearly defined guidelines, and lastly, the clinical course of the disease is difficult to predict. In order to minimize colectomy failure in patients with severe CDI, many providers advocate early surgical intervention, which is associated with an increased rate of operative intervention and survival [21].

However, early TCEI in CDI is far from life-saving with a recent meta-analysis revealing a $30 \mathrm{~d}$ mortality rate of $41.3 \%$ [22]. This stark reality has led some investigators to employ earlier operative intervention in an attempt to mitigate the severe CDI-associated mortality rates. One small series incorporated early diverting loop ileostomy with vancomycin colonic lavage and found a $30 \%$ reduction in post-operative mortality rates compared with historical TCEI control patients [23]. Although these results sound promising, a more recent meta-analysis found that when TCEI was not performed, reoperation to resect further bowel was necessary in $16 \%$ of patients [22]. The authors conclude that TCEI should be the primary surgical treatment for patients with severe CDI. This notion goes hand in hand with the premise that less extensive surgery may have a role in selected patients with earlier-stage CDI; however, the supporting data are sparse at best [24].

Given the importance of rapid source control via TCEI for fulminant CDI, it is important to understand that continued management of any remaining infected colonic tissue must occur. In order to do this, current recommendations suggest 
the use of oral and rectal vancomycin to eradicate any potential continued source of infection $[25,26]$.

\section{Conclusion}

Severe CDI should have early surgical consult. Indications for surgical intervention remain poorly defined. Subtotal colectomy with end ileostomy appears superior to partial colectomy and other surgical approaches in regards to the need for subsequent re-operation. Although loop ileostomy with colonic washout is promising, data remain limited and ability to define full extent of necrosis may be limited.

\section{Acknowledgment}

This work was supported by R01GM078054 from the National Institute of General Medical Sciences.

\section{Author Disclosure Statement}

No competing financial interests exist.

\section{References}

1. Larson HE, Price AB, Honour P, Borriello SP. Clostridium difficile and the aetiology of pseudomembranous colitis. Lancet 1978;1:1063-1066.

2. Loo VG, Poirier L, Miller MA, et al. A predominantly clonal multi-institutional outbreak of Clostridium difficileassociated diarrhea with high morbidity and mortality. $\underline{\mathrm{N}}$ Engl J Med 2005;353:2442-2449.

3. Gerding DN, Johnson S, Peterson LR, et al. Clostridium difficile-associated diarrhea and colitis. Infect Control Hosp Epidemiol 1995;16:459-477.

4. McDonald LC, Owings M, Jernigan DB. Clostridium difficile infection in patients discharged from US short-stay hospitals, 1996-2003. Emerg Infect Dis 2006;12:409-415.

5. Kuijper EJ, Coignard B, Brazier JS, et al. Update of Clostridium difficile-associated disease due to PCR ribotype 027 in Europe. Euro Surveill 2007;12:E1-E2.

6. Cohen SH, Gerding DN, Johnson S, et al. Clinical practice guidelines for Clostridium difficile infection in adults: 2010 update by the society for healthcare epidemiology of America (SHEA) and the infectious diseases society of America (IDSA). Infect Control Hosp Epidemiol 2010;31: 431-455.

7. Deneve C, Janoir C, Poilane I, et al. New trends in Clostridium difficile virulence and pathogenesis. Int J Antimicrob Agents 2009;33:S24-S28.

8. Merrigan M, Venugopal A, Mallozzi M, et al. Human hypervirulent Clostridium difficile strains exhibit increased sporulation as well as robust toxin production. J Bacteriol 2010;192:4904-4911.

9. Makris AT, Gelone S. Clostridium difficile in the long-term care setting. J Am Med Dir Assoc 2007;8:290-299.

10. Zilberberg MD, Nathanson BH, Sadigov S, et al. Epidemiology and outcomes of Clostridium difficile-associated disease among patients on prolonged acute mechanical ventilation. Chest 2009;136:752-758.

11. Aseeri M, Schroeder T, Kramer J, Zackula R. Gastric acid suppression by proton pump inhibitors as a risk factor for Clostridium difficile-associated diarrhea in hospitalized patients. Am J Gastroenterol 2008;103:2308-2313.
12. Goodhand JR, Alazawi W, Rampton DS. Systematic review: Clostridium difficile and inflammatory bowel disease. Aliment Pharmacol Ther 2011;33:428-441.

13. Hookman P, Barkin JS. Clostridium difficile associated infection, diarrhea and colitis. World J Gastroenterol 2009; 15:1554-1580.

14. Schaier M, Wendt C, Zeier M, Ritz E. Clostridium difficile diarrhoea in the immunosuppressed patient-update on prevention and management. Nephrol Dial Transplant 2004;19:2432-2436.

15. Garey KW, Sethi S, Yadav Y, DuPont HL. Meta-analysis to assess risk factors for recurrent Clostridium difficile infection. J Hosp Infect 2008;70:298-304.

16. Johnson S. Recurrent Clostridium difficile infection: A review of risk factors, treatments, and outcomes. $\mathrm{J}$ Infect 2009;58:403-410.

17. Zilberberg MD, Shorr AF, Micek ST, et al. Clostridium difficile-associated disease and mortality among the elderly critically ill. Crit Care Med 2009;37:2583-2589.

18. Pepin J, Vo TT, Boutros M, et al. Risk factors for mortality following emergency colectomy for fulminant Clostridium difficile infection. Dis Colon Rectum 2009;52:400-405.

19. Wang MF, Ding Z, Zhao J, et al. Current role of surgery for the treatment of fulminant Clostridium difficile colitis. Chin Med J (Engl) 2013;126:949-956.

20. Lamontagne F, Labbé AC, Haeck O, et al. Impact of emergency colectomy on survival of patients with fulminant Clostridium difficile colitis during an epidemic caused by a hypervirulent strain. Ann Surg 2007;245:267-272.

21. Sailhamer EA, Carson K, Chang Y, et al. Fulminant Clostridium difficile colitis: Patterns of care and predictors of mortality. Arch Surg 2009;144:433-439.

22. Bhangu A, Nepogodiev D, Gupta A, et al. Systematic review and meta-analysis of outcomes following emergency surgery for Clostridium difficile colitis. Br J Surg 2012; 99:1501-1513.

23. Neal MD, Alverdy JC, Hall DE, et al. S. Diverting loop ileostomy and colonic lavage: An alternative to total abdominal colectomy for the treatment of severe, complicated Clostridium difficile associated disease. Ann Surg 2011;254: 423-427.

24. Seltman AK. Surgical management of Clostridium difficile colitis. Clin Colon Rectal Surg 2015;25:204-209.

25. Erikstrup LT, Aarup M, Hagemann-Madsen R, et al. Treatment of Clostridium difficile infection in mice with vancomycin alone is as effective as treatment with vancomycin and metronidazole in combination. BMJ Open Gastroenterol 2015;2:156-162.

26. Van der Wilden GM, Subramanian MP, Chang Y, et al. Antibiotic regimen after a total abdominal colectomy with ileostomy for fulminant Clostridium difficile colitis: A multi-institutional study. Surg Infect 2015;16:455-460.

Address correspondence to:

Dr. Joseph Cuschieri

Department of Surgery

University of Washington 325 9th Avenue

Seattle, WA 98104-2499

E-mail: jcushie@uw.edu 